\title{
Semantic Adaptation of Multimedia Documents
}

\author{
Azze-eddine Maredj \\ Research Center on Scientific \\ and Technical Information \\ Ben Aknoun Algiers, Algeria
}

\author{
Noureddine Tonkin \\ Research Center on Scientific \\ and Technical Information \\ Ben Aknoun Algiers, Algeria
}

\begin{abstract}
A multimedia document should be presented on different platforms therefore, the adaptation of its content is necessary. In this contribution, we give some proposals to improve and extend the semantic approach based on conceptual neighborhood graphs in order to best preserve the proximity between the adapted and the original documents and to deal with models that define delays and distances.
\end{abstract}

\section{Introduction}

The ongoing developments in communication technology and computing systems make the communication dominated by multimedia data. The heterogeneous nature of the devices used to access the multimedia content and the user preferences makes it necessary to adapt the documents. A lot of works have been undertaken in the multimedia documents adaptation and we can group them into four categories: (i) specification of alternatives, (ii) using transformation rules, (iii) using flexible models and (iv) semantic and dynamic approaches. In the approaches based on alternatives specification and transformation rules, the adaptation is explicit: the author knows all the target profiles and then, specifies directly the different versions of his document according to those profiles. In this case, it's extremely difficult and hard to foresee all the possibilities in advance. In the systems based on flexible models, the adaptation (when it is not limited to the target material and to the sequencing of the media) depends mostly on the description languages and standards. Another approach proposed in [17], tries to overpass those issues by using the relations specification and the conceptual neighborhood graphs (CNG) of the relations [10] to search the substitution relations of the ones that do not comply with the target profile (device capabilities and user preferences). However, those relations are not most often replaced by the closest, due to the fact that the weights of the arcs of the CNG are set to one (1). This means that a relation can be replaced by any one of its neighbors. Moreover, the relations of the used models (temporal and spatial) do not consider the delays and the distances whereas the documents are generally produced using more complex models to insure a high expressiveness level, a highly prized quality in the multimedia document authoring systems.

In this paper, we propose an extension of the latter approach to insure greater proximity between the adapted and the initial documents and to deal with models that define delays and distances. To situate our contribution, we present in section 2 the similar works. In section 3 , we present the extension of the CNG that permits us to best preserve the similarity between the adapted and the initial documents and to deal with more complex relations models. To validate our proposition, we present in section 4 the adaptation procedure and an illustrating example of our contribution. Section 5 concludes this paper and gives some perspectives.

\section{Related works}

Several approaches have been proposed for the multimedia documents adaptation and we group them into four categories.

\subsection{Specification of alternatives}

The author of the document specifies a set of presentation alternatives by defining criteria on some media. If the media satisfy the criteria then, they are selected and presented else, they are deleted from the presentation. In this category, we can find three types of approaches.

The first type is called beforehand adaptation. The author specifies different versions of the document according to the target profiles. SMIL is an example, it uses the operator switch to specify the alternatives that are played only if they comply with the target profile.

The second type is the a posteriori adaptation where the adaptation is based on the content of the presentation. The author uses annotations to identify the pertinent parts of his document and then, a filter is applied to the document to select the parts that comply with the established selection criteria. The language proposed in [20] is an example. It is based on a temporal extension of HTML that offers new tags and attributes. The adaptation is done by 
applying style sheets as proposed in [22] like XSLT for XML documents and CSS for HTML document. We can also mention the WebView system [9] that uses extension of XPath to select parts of the document.

The last type concerns the hybrid approaches that merge the two precedent types. The author does not only use the specification of alternatives according to the different profiles but also adds annotations to the document. The AHM model (Amsterdam Hypermedia Model) [15] is an example. It is constructed by combining the Dexter Model [13] [14] with the CMIF model [7] to which were added some extensions concerning the hypermedia.

The advantage of this family is that the adaptation is instantly and flexible in some cases. However, the author has to foresee all the possible adaptation constraints (target profiles), specify all the conceivable alternatives and perform an extra effort for the annotation. Furthermore, the selection conditions are strongly dependent on the used annotation language.

\subsection{Using transformation rules}

This category uses a set of transformation rules that are applied to the multimedia documents. The adaptation consists on selecting and applying rules to transform the document to satisfy the target profile. For this category, the software infrastructure NAC (Negotiation and Adaptation Core) [18] is the most representative. It defines components that take part in the negotiation and the content adaptation process. NAC provides two adaptation types: structural adaptation and media adaptation. For the first type, NAC uses the transformation language XSLT. However, to avoid the definition of a transformation sheet for each target profile and for each document, generic templates are defined. Nevertheless, all the transformations should be specified in the transformation rules base. We can name also, the MPEG-21 standard [5], Xadaptor [16] and AHA [6]. The advantage of this approach is that the author has not to care about the execution context of his document. Furthermore, these rules can be completed if new contexts appear. However, the entire transformation rules should be specified to ensure an efficient adaptation.

\subsection{Flexible documents models}

The adapted document is generated automatically from a non-composed set of media represented by a model defining an abstraction of the document. Thanks to a formatting model, a multimedia presentation may be generated. The adaptation constraints of a target profile may be found at two different levels: at the abstraction level and at the document generation level. In this category, we can find four (04) models.

The first model uses the concept of Customizable virtual document (CVD) [21]. The virtual document is considered as customizable if the document composition methods permit its adaptation for a given reader.

The second type Guypers [11], aims to generate web-based presentation for multimedia databases. It uses the semantic relations between media and ontologies [12]. The aim of this model is to generate multimedia documents to meet the capabilities of the used platform.

The third model is MM4U [4]. It uses different modules related to the user profile, the multimedia data, the media composition and the document generation. Then, the multimedia document specification can be adapted according to the execution context and generated in different formats like SMIL or HTML. The inconvenient is that the framework uses proprietary languages.

The fourth model, STAMP (Synchronized Templates for Adaptable Multimedia Presentations) [3] aims to dynamically generate multimedia presentations from semi-structured content. This approach is based on the construction of templates describing the spatial, the temporal and the hypermedia organizations where the content may vary. During the generation of the final presentation, the presentation model is adapted automatically when it does not satisfy the constraints of the target profile. However, the adaptation is limited to the exclusion of media or the sequencing of the media that cannot be played simultaneously.

\subsection{Semantic and dynamic approaches}

In [17], an approach based on the qualitative specifications of the document was proposed. This approach extends the temporal adaptation approach proposed in [8] to the spatial and hypermedia dimensions. Each document is considered as a set of potential executions and each profile is considered as a set of possible executions. The adaptation is done according to the context at the execution time. It consists on calculating the intersection between the potential (initial specification models) and the possible executions corresponding to the target profile. Its advantage is it does not restrict the profile constraints and its independence from description languages. However, the use of the CNG where all the weights of the arcs are set to one (01) assumes that a relation may be replaced by any one of its immediate neighbors while there are substantial differences between them; especially when using complex relations models. Furthermore, in the used model [1] the delays and the distances are not considered. 


\section{Proposed approach}

In the proposed approach, we use the CNG of the relations proposed in [10] and used in [8] and [17]. To best preserve the proximity between the adapted document and the initial document and to deal with models that define delays and distances, we propose a new construction of the CNG. In the reminder of this paper, we use the Wahl and Rothermel model [23] for the temporal relations and the directional model [19] for the spatial relations.

\subsection{Weighting of the CNG}

In [8] and [17], the used model is the Allen's model and the weights assigned to the arcs of the CNG are set to one (01) (figure 1). In order to replace a relation that does not comply with a profile by the closest one, we propose a new weighting of the CNG. For this, we start by identifying all the information items characterizing a relation which will serve as criteria of the comparison between the relations.

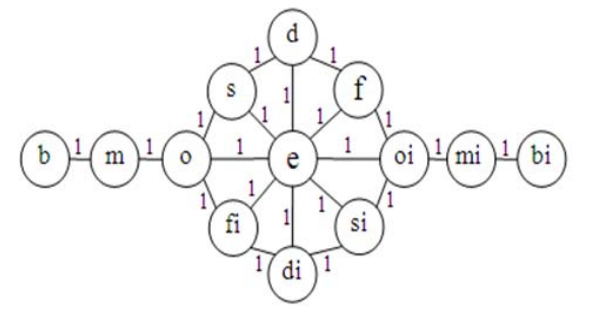

Figure1. CNG of Allen relations

\subsubsection{Information elements of a relation}

The analysis of a relation between two media A and $\mathrm{B}$ (Figure 2) on a time axis showed that the positioning is done according to the values and the order (precedes $(>)$, succeeds $(<)$ or equal $(=)$ ) of their respective edges (beginning and ending instants).

Table 1 gives the 16 selected information items that characterize a relation. For each relation, we attribute the value one (01) when the information is contained and zero (0) elsewhere. Table 2 gives the 16 information items of the Wahl and Rothermel model relations.

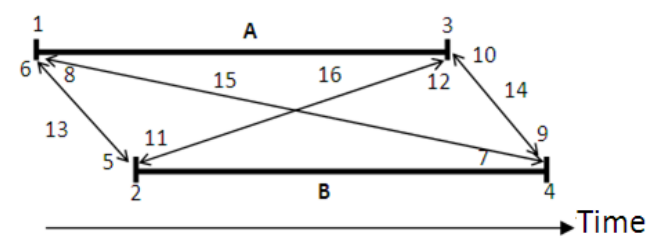

Figure 2. Information of a relation
Table 1. Information characterizing a relation

\begin{tabular}{|l|l|l|l|l|l|l|l|l|}
\hline Information & $\mathbf{1}$ & $\mathbf{2}$ & $\mathbf{3}$ & $\mathbf{4}$ & $\mathbf{5}$ & $\mathbf{6}$ & $\mathbf{7}$ & $\mathbf{8}$ \\
\hline Signification & $\mathrm{b}(\mathrm{A})$ & $\mathrm{b}(\mathrm{B})$ & $\mathrm{e}(\mathrm{B})$ & $\mathrm{e}(\mathrm{B})$ & $1>2$ & $1<2$ & $1>4$ & $1<4$ \\
\hline Information & $\mathbf{9}$ & $\mathbf{1 0}$ & $\mathbf{1 1}$ & $\mathbf{1 2}$ & $\mathbf{1 3}$ & $\mathbf{1 4}$ & $\mathbf{1 5}$ & $\mathbf{1 6}$ \\
\hline Signification & $3>4$ & $3<4$ & $3>2$ & $3<2$ & $1=2$ & $3=4$ & $1=4$ & $3=2$ \\
\hline
\end{tabular}

Table 2. Information of the temporal relations

\begin{tabular}{|l|l|l|l|l|l|l|l|l|l|l|l|l|l|l|l|l|}
\hline & 1 & 2 & 3 & 4 & 5 & 6 & 7 & 8 & 9 & 10 & 11 & 12 & 13 & 14 & 15 & 16 \\
\hline Before & 0 & 1 & 1 & 0 & 0 & 1 & 0 & 1 & 0 & 1 & 0 & 1 & 0 & 0 & 0 & 0 \\
\hline Overlaps & 1 & 1 & 1 & 1 & 0 & 1 & 0 & 1 & 0 & 1 & 1 & 0 & 0 & 0 & 0 & 0 \\
\hline Endin & 0 & 1 & 1 & 1 & 0 & 1 & 0 & 1 & 0 & 1 & 1 & 0 & 0 & 0 & 0 & 0 \\
\hline Cobegin & 1 & 1 & 0 & 0 & 0 & 1 & 0 & 1 & 0 & 1 & 1 & 0 & 0 & 0 & 0 & 0 \\
\hline Coend & 0 & 0 & 1 & 1 & 0 & 1 & 0 & 1 & 0 & 1 & 1 & 0 & 0 & 0 & 0 & 0 \\
\hline Beforeendof $^{-1}$ & 0 & 1 & 1 & 0 & 0 & 1 & 0 & 1 & 0 & 1 & 1 & 0 & 0 & 0 & 0 & 0 \\
\hline Cross $^{-1}$ & 1 & 1 & 1 & 1 & 0 & 1 & 0 & 1 & 0 & 1 & 1 & 0 & 0 & 0 & 0 & 0 \\
\hline Delayed $^{-1}$ & 1 & 1 & 1 & 1 & 0 & 1 & 0 & 1 & 0 & 1 & 1 & 0 & 0 & 0 & 0 & 0 \\
\hline Startin $^{-1}$ & 1 & 1 & 1 & 0 & 0 & 1 & 0 & 1 & 0 & 1 & 1 & 0 & 0 & 0 & 0 & 0 \\
\hline While $^{-1}$ & 1 & 1 & 1 & 1 & 1 & 0 & 0 & 1 & 0 & 1 & 1 & 0 & 0 & 0 & 0 & 0 \\
\hline Contains $^{1}$ & 1 & 1 & 1 & 1 & 0 & 1 & 0 & 1 & 1 & 0 & 1 & 0 & 0 & 0 & 0 & 0 \\
\hline Beforeendof $^{1}$ & 1 & 0 & 0 & 1 & 1 & 0 & 0 & 1 & 1 & 0 & 1 & 0 & 0 & 0 & 0 & 0 \\
\hline Cross $^{1}$ & 1 & 1 & 1 & 1 & 1 & 0 & 0 & 1 & 1 & 0 & 1 & 0 & 0 & 0 & 0 & 0 \\
\hline Delayed $^{1}$ & 1 & 1 & 1 & 1 & 1 & 0 & 0 & 1 & 1 & 0 & 1 & 0 & 0 & 0 & 0 & 0 \\
\hline Startin $^{1}$ & 1 & 1 & 0 & 1 & 1 & 0 & 0 & 1 & 1 & 0 & 1 & 0 & 0 & 0 & 0 & 0 \\
\hline Cobegin-1 $^{-1}$ & 1 & 1 & 0 & 0 & 1 & 0 & 0 & 1 & 1 & 0 & 1 & 0 & 0 & 0 & 0 & 0 \\
\hline Endin $^{-1}$ & 1 & 0 & 1 & 1 & 1 & 0 & 0 & 1 & 1 & 0 & 1 & 0 & 0 & 0 & 0 & 0 \\
\hline Coend $^{-1}$ & 0 & 0 & 1 & 1 & 1 & 0 & 0 & 1 & 1 & 0 & 1 & 0 & 0 & 0 & 0 & 0 \\
\hline Overlaps $^{-1}$ & 1 & 1 & 1 & 1 & 1 & 0 & 0 & 1 & 1 & 0 & 1 & 0 & 0 & 0 & 0 & 0 \\
\hline Before $^{-1}$ & 1 & 0 & 0 & 1 & 1 & 0 & 1 & 0 & 1 & 0 & 1 & 0 & 0 & 0 & 0 & 0 \\
\hline
\end{tabular}

\subsubsection{Calculation of the similarity degree between relations}

To calculate the similarity degree between a relation $r$ and its immediate neighbor $r$, we wse the Manhattan distance defined as follows: $d\left(r, r^{\prime}\right)=$ $\sum_{i=1}^{16}\left|v_{i}-u_{i}\right|$ where $v_{i}$ and $u_{i}$ are respectively the 16 information items of $r$ and r'. Table 3 gives the distances between each relation and its neighbors.

Table 3. Distances between the relations and theirs neighbors

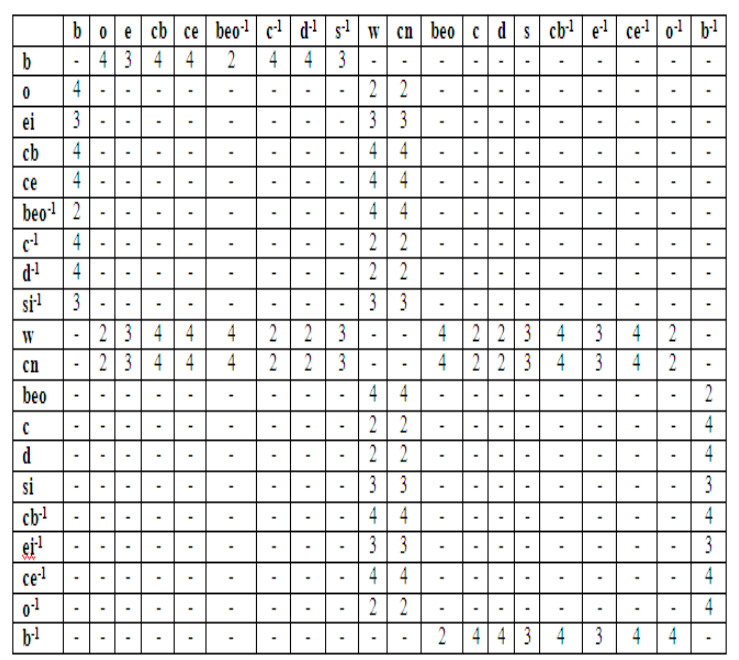




\subsection{Relations models with delays and distances}

Generally, in authoring multimedia systems the used models define delays and distances to enhance their expressiveness degree. Therefore, our approach should deal with these models. For this, we replace each node of the CNG by a graph called relaxation graph (RG). It is constituted by the different forms that a relation can take: considering the delays (distances) specification as a strong constraint, those forms are obtained by relaxing this constraint to a weaker constraint. For instance, the relaxation of A before (5) B would be A before (-) B. The "-" character represent a relaxation status, where its value, other than 5 , is to be determined by the constraints solver (in our case, we use the Cassowary Solver [2]).

The RG is obtained by a progressive relaxation of the delays (distances). Figure 3 gives the RG of the relations with one, two (maximum number of distances) and three (maximum number of delays).

Thus, the relaxation may lead to an adaptation solution without replacement of the relations that do not comply with the target profile; consequently the adapted document will be as close as possible to the initial document.

- Relation with one delay

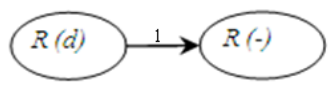

- Relation with two delays

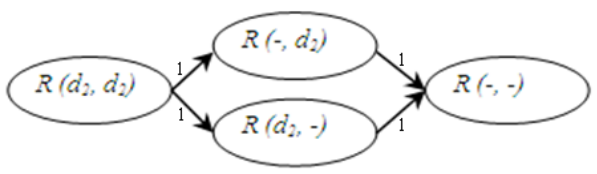

- Relation with three delays

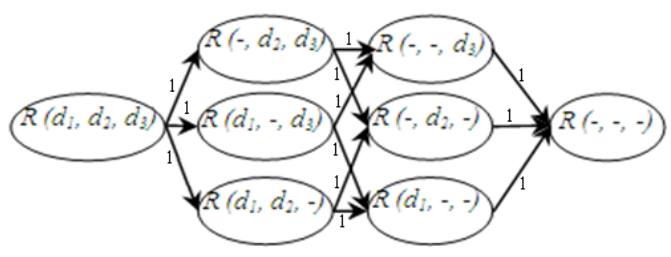

Figure 3. Relaxation graphs

Since the relaxation order does not affect the proximity of a relation with its neighbors in the RG, we set the weights of the arcs to one (01).

\subsection{CNG construction and traversal}

To determine the neighbors of a relation, we start by elaborating the CNG as proposed in [10] then; we replace each node (relation) by its corresponding GR.
The graph traversal starts from the RG of the relation to be replaced. According to delays (distances) of that relation, we identify the corresponding node in the RG then, we traverse the graph from this node. Once the ending node of the RG (node where all the delays (distances) are relaxed) is achieved, we move to the node of the following RG having the maximum of similar delays (distances) as in the relation to be replaced. For instance, to replace the relation A Overlaps $(5,8,10)$ $\mathrm{B}$ by the relation Delayed-1, we move directly to the node of the form Delayed-1 $(5,10)$. Table 4 gives the destination nodes of the relation Overlaps (d1, d2, d3). The same principle is applied for the other relations.

Table 4. Destination Nodes of the relation Overlaps

\begin{tabular}{|c|c|}
\hline Relation & Needs destination \\
\hline Overlaps $\left(\mathrm{d}_{1}, \mathrm{~d}_{2}, \mathrm{~d}_{3}\right)$ & $\begin{array}{l}\text { Before }(-) \\
\text { Cobegin }\left(\mathrm{d}_{1}\right) \\
\text { Endin }\left(\mathrm{d}_{2}, \mathrm{~d}_{3}\right) \\
\text { Coend }\left(\mathrm{d}_{3}\right) \\
\text { Beforeendof }{ }^{-1}\left(\mathrm{~d}_{2}\right) \\
\text { Cross }{ }^{-1}\left(-, \mathrm{d}_{2}\right) \\
\text { Delayed } \\
\text { Startin }^{-1}\left(\mathrm{~d}_{1}, \mathrm{~d}_{3}\right) \\
\text { While }\left(-, \mathrm{d}_{3}\right) \\
\text { Contains }\left(\mathrm{d}_{1},-\right) \\
\text { Beforeendof }(-) \\
\text { Cross }\left(-, \mathrm{d}_{2}\right) \\
\text { Delayed }(-,-) \\
\text { Startin }(-,-) \\
\text { Cobegin } \\
\text { Endin }^{-1}(-) \\
\text { Coend }(-,-) \\
\text { Overlaps }(-) \\
\text { Before }^{-1}(-)\end{array}$ \\
\hline
\end{tabular}

\subsubsection{CNG of the temporal relations}

We present in the figure 4, the proposed CNG of the Wahl and Rothermel temporal relations obtained by following the proposed approach.

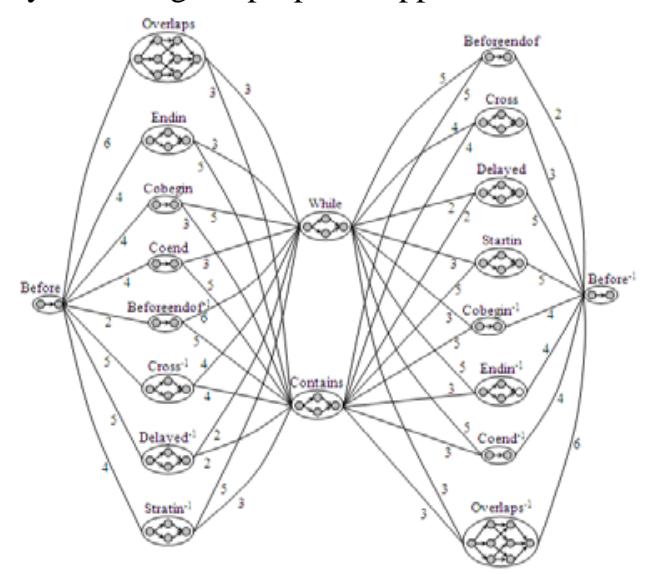

Figure 4. The CNG of the Wahl and Rothermel model relations 


\subsubsection{CNG of the spatial relations}

In the directional model of the spatial relations [19], a media is represented by two intervals corresponding to its projections on the horizontal and vertical axes. To homogenize the temporal and the spatial representation and to have a high integration level of the two dimensions (spatial and temporal) treatment, the spatial relations are represented by using the temporal relations obtained by the combination of the media intervals on the horizontal and the vertical axes. There are 20 relations (Wahl and Rothermel model) between two intervals on each axis, which gives us 202 possible relations between two media. For instance, the relation $\mathrm{A}$ left $\mathrm{B}$ is transformed to the two relations A before (d1) B (on the horizontal axis) and A While (d2, d3) B (on the vertical axis). Consequently, the CNG of the spatial relations is obtained by the square product of the CNG of temporal relations.

\subsubsection{Conceptual neighborhood graph of the hypermedia relations}

For the same integration purpose, we consider a hypermedia link as a media as well as any other media of the document. In figure 5 , the media image has a hypermedia link (the dotted area) to the media text. It is enabled from the tenth second of the media image to its end. This link can be specified by the temporal relation link while $(10,0)$ image and the spatial relation link (while $(10,10)$, while $(50,0)$ ) image.

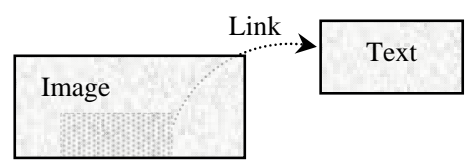

\section{Figure 5. Hypermedia link between two media}

Therefore, the same adaptation procedure of the temporal and spatial dimensions can be applied to the hypermedia adaptation. However, as a hypermedia link is spatially and temporally included in its anchor, only the relations of inclusion can be used. Thus, we perform the hypermedia adaptation using solely the relaxation of the relation while.

\subsection{Adaptation procedure}

The semantic adaptation of multimedia documents is achieved by modifying the specification of the initial document. This involves finding another set of solutions satisfying the constraints of the target profile. This set of relations is obtained by combining the candidate relations to the replacement of each relation of the initial document.
The adapted document will correspond to the coherent solution with the smallest conceptual distance [17] defined as follows: $\mathrm{dc}(\mathrm{sol}) \mathrm{k}$ $=\sum_{i=1}^{n} d c(r i, r j)$ with $\mathrm{n}$ is the document relations number, $r j$ is a candidate relation to the replacement of the relation ri and dc(ri,rj) is the conceptual distance ri between and rj.

For the temporal relations, dc(ri,rj) is calculated by traversing the CNG and searching for the shortest path between the two relations. For instance, dc(Before(-), while (-, -)) = dc (before (-), endin(-)) + dc (endin(-), while $(-,-))=4+3=7$

For a spatial relation, the conceptual distance is given by the sum of the conceptual distances between the horizontal components and vertical components of the two relations. For instance, for the two relations $\mathrm{ri}=\{$ before, cobegin $\}$ and $r j=\{$ endin, while $\}$, we have $\mathrm{dc}(\mathrm{ri}, \mathrm{rj})=\mathrm{dcx}(\mathrm{ri}, \mathrm{rj})+$ $\mathrm{dcy}(\mathrm{ri}, \mathrm{rj})=\mathrm{dc}($ before,endin $)+\mathrm{dc}($ cobegin, while $)=4+5$ $=9$.

\subsection{Adaptation algorithm}

Input : $\mathrm{MI}_{1 \mathrm{j}}$ : //Matrix of the document relations

$/ /$ replacement relations search

For $\mathrm{i}=0$ to $\mathrm{n}-1$ do $/ / \mathrm{n}$ number of media

for $\mathrm{j}=0$ to $\mathrm{n}-1$ do

For $\mathrm{k}=1$ to NR do // NR: number of the relations of the model

if respecteProfil $(\mathrm{Rm}[\mathrm{k}])$ then $/ / \mathrm{Rm}$ set of the model relations

MS [i, j] $\leftarrow$ MS [i, j] $\cup\{\mathrm{Rm}[\mathrm{k}]\}$;

End if

End for

End for

End for

// Elaboration of the possible combinations

//output : combinations list $C_{p}$

$\mathrm{C}_{\mathrm{p}}=$ ElaborateCombinationsMatrix $\left(\mathrm{MS}_{\mathrm{ij}}\right)$;

// Sort combinations according to the conceptual distance

For $\mathrm{i}=0$ to nCombinations -1 do

$\mathrm{d}[\mathrm{i}] \leftarrow 0$;

// Matrix of the

conceptual distances

$$
\begin{aligned}
& \text { For } \mathrm{j}=0 \text { to } n-1 \text { do } \\
& \quad d[i]=d[i]+\text { Djikstra(C[i,j], MR[i,j]); } \\
& \text { Endfor }
\end{aligned}
$$

Endfor

QuickSortCombinations(C[i], d[i]);

// Consistency Verification

found $\leftarrow$ false ;

For $\mathrm{i}=0$ to nCombinations -1 do

if Consistency (C[i]) Then

Solution $\leftarrow(\mathrm{C}[\mathrm{i}])$;

found $\leftarrow$ true ; Break ; 
End if

End for

If found = false Then Write ('no adaptation ');

The algorithm takes as input the matrix MIij: matrix of the complete relations graph of the initial specification (figure 5.b for example). Once the substitution matrix MSij, which gives for each relation of the matrix Mij the relations candidates for its substitution from those that meet the target profile constraints among the relations of the model, is identified, we determine by combinations, all the possible solutions Ci from the matrix MSij. Next, we perform an ascending sort of all solutions of $\mathrm{Ci}$ with the classical sorting algorithm "quick sort" using the conceptual distances calculated by using the Dijkstra's shortest path algorithm. This will ensure that the solutions are sorted from the closest specification to the farthest from the original. Finally, we call the constraints solver (cassowary [2]) for the consistency verification and the calculation of the solution for each specification in the order defined by the sort. The first verification that gives a consistency stops the process and the adapted document is generated.

\subsection{An adaptation example}

Let us consider the initial multimedia document of figure 6 and the following profile:

Media should not start at the same time.

Streaming media cannot be played simultaneously.

For this example, we take into consideration only the temporal aspect. The same treatment is valid for the spatial and the hypermedia aspects.

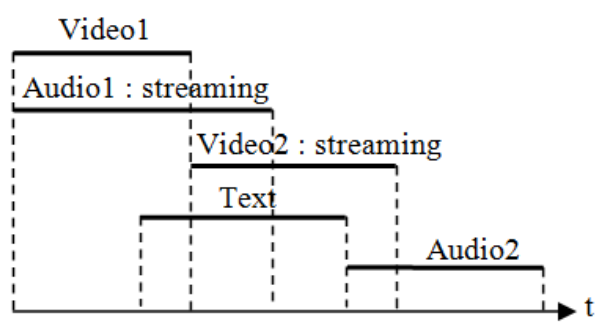

a. Temporal representation

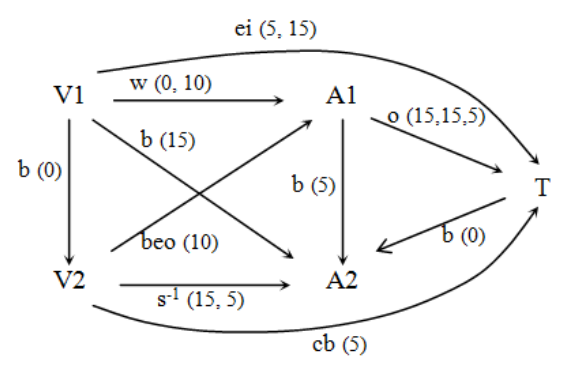

b. Relations graph
The relations video 1 while $(0,10)$ audio 2 and video2 beforeendof (-) audio1 do not meet the target profile constraints. For that, the document must be adapted. After the execution of the adaptation algorithm, we obtain the solution given in figure 7 . Table 5 summarizes all the performed modifications applied to the relations and gives an idea on the proximity of the solution and the initial document.

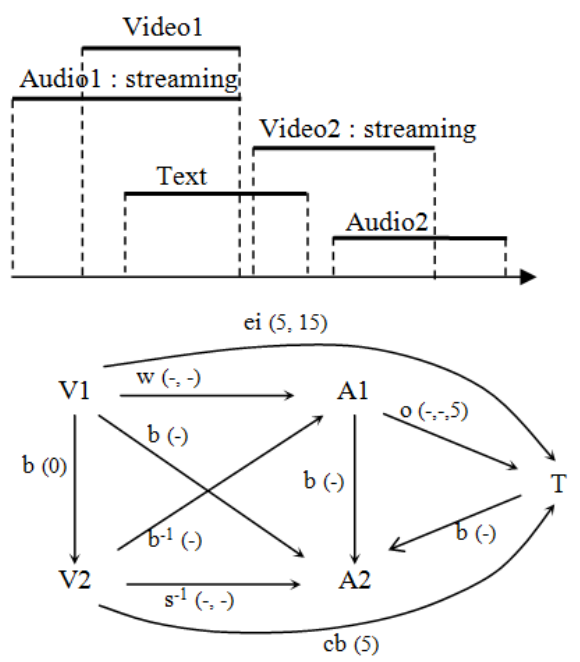

Figure 7. The adapted document

Table 5. Relations modification

\begin{tabular}{|l|c|l|}
\hline Initial specification & Adaptation solution & Action \\
\hline V1 w (0, 10) A1 & V1 w (-, -) A1 & Relaxation \\
\hline V1 b (0) V2 & V1 b (0) V2 & No change \\
\hline V1 e (5, 15) T & V1 e (5, 15) T & No change \\
\hline V1 b (15) A2 & V1 b (-) A2 & Relaxation \\
\hline V2 beo (10) A1 & V2 b-1 (-) A1 & Replacement \\
\hline A1 o (15, 15, 5) T & A1 o (-, -, 5) T & Relaxation \\
\hline A1 b (5) A2 & A1 b (-) A2 & Relaxation \\
\hline V2 cb (5) T & V2 cb (5) T & No change \\
\hline V2 s-1 (15, 5) A2 & V2 s-1 (-, -) A2 & Relaxation \\
\hline T b (0) A2 & T b (-) A2 & Relaxation \\
\hline
\end{tabular}

\section{Conclusion}

In this paper, we proposed a semantic approach of the temporal, spatial and hypermedia adaptation of multimedia documents. The originality of this approach is, from one side, that the adapted document is semantically as close as possible to the initial document, and from the other side, the extension of the conceptual neighborhood graph permits to deal with models that use delays and distances.

We have shown how to best differentiate the similarity degree of the relations by proposing a new way of building the conceptual neighborhood graph and we introduced the concept of the relation relaxation that permit to keep the relations or

Figure 6. Initial Document 
otherwise to replace them while maintaining the maximum number of delays (distances).

Moreover, as the objective is primarily to best preserve the message intended by the author of the initial document (Otherwise what is the benefit of such an adaptation?), it seems quite rightful to ask the following question: Is it appropriate to deliver the adapted document even though the message of the initial document may be changed? Otherwise what are the parameters should be taken into account?

The first direction of our future work would be to determine a similitude measure between the adapted document and the initial one by using some extra information (annotations) like relations weights in the document to determine the relations to be modified or deleted if it's necessary.

\section{References}

[1] Allen J. F., "Maintaining knowledge about temporal intervals", Communications of the ACM, vol. 26 no. 11, pp. 832-843, 1983.

[2] Badros G. J. et al, "The Cassowary linear arithmetic constraint solving algorithm", ACM Transactions on Computer-Human Interaction (TOCHI), vol. 8, no. 4, pp. 267-306, 2001.

[3] Bilasco I. M. et al,’STAMP: a model for generating adaptable multimedia presentations", Multimedia Tools and Applications, Special Issue on Metadata and Adaptibility in Web-based Information Systems, vol. 25, no. 3 pp. 361-375, 2005.

[4] Boll S., "MM4U : a framework for creating personalized multimedia content. In Proceeding of the ninth International Conference on Distributed Multimedia Systems, Miami, USA, pp. 12-16, 2003.

[5] Jan Bormans et Keith Hill: MPEG-21 Overview v.5. ISO/IECJTC1/SC29/WG11/N5231, 2002. http://www.chiariglione.org/mpeg/standards/mpeg21/mpeg-21.htm

[6] De Bra P. et al, “ The design of AHA! In U_e KockWiil, Peter J. Nurnberg et Jessica Rubart, éditeurs : Proceedings of the 17thACM Conference on Hypertext and Hypermedia, pages 133-134. ACM Press, 2006.

[7] Bulterman D. et al, "A structure for transportable dynamic multimedia documents", In Proceedings of the Summer 1991 Usenix Conference, Nashville, Tennessee, USA, pp. 137-156, 1991.

[8] Euzenat J. et al, “A semantic framework for multimedia document adaptation”, in proceedings of the 18th International Joint Conference on Artificial Intelligence (IJCAI). Acapulco, Mexico, pp. 31-36, 2003.

[9] Juliana F. et al, "WebViews : accessing personalized web content and services". In Proceedings of the 10th international conference on World Wide Web, Hong Kong, pp. 576-586, 2001.

[10] Freksa C., "Conceptual neighborhood and its role in temporal and spatial reasoning”, In Proceedings of the IMACS Inter. Work. on Decision Support Systems and Qualitative Reasoning. Toulouse, France, 181-187, 1991.

[11] Geurts J. et al, “Application specific constraints for multimedia presentation generation", In Proceedings of the 8th International Conference on Multimedia Modeling, Amsterdam, The Netherlands, pp. 247-266, 2001.

[12] Geurts J. et al, "Towards ontology-driven discourse : From semantic graphs to multimedia presentations", In Proceedings of the 2nd International Semantic Web Conference, Sanibel Island, Florida, USA, pp. 597-612, 2003.

[13] Gronbaek K., Randall H, “Trigg: Design issues for a Dexter-based hypermedia system”, Communications of the ACM, vol. 37, no. 2, pp. 40-49, 1994.

[14] Halasz F., Schwartz M., "The Dexter hypertext reference model". Communications of the ACM, vol. 37, no. 2, pp. 30-39, 1994.

[15] Hardman L. et al, "The Amsterdam hypermedia model : adding time and context to the dexter model”, Communications of the ACM, vol. 37, no. 2, pp. 50-62, 1994.

[16] He J. et al, "A flexible content adaptation system using a rule-based approach”, IEEE Transactions on Knowledge and Data Engineering, vol. 19, no. 1, pp. 127140, 2007.

[17] Laborie S., et al "Semantic Adaptation of Multimedia Documents". Multimedia Tools and Applications (MTAP), pages 1-20, juin 2010.

[18] Lemlouma T., Layaïda N., "The negotiation of multimedia content services in heterogeneous environments", In Proceedings of the 8th International Conference on Multimedia Modeling, Amsterdam, The Netherlands, pp. 187-206, 2001.

[19] Papadias D. and Sellis T, “Qualitative Representation of Spatial Knowledge in Two-Dimensional Space”, Special Issue on Spatial atabases, vol. 3, no. 4, pp. 479-516, 1994.

[20] Rousseau F., et al, "User adaptable multimedia presentations for the world wide web”, In Proceedings of the 8th International Conference on World Wide Web, Elsevier North-Holland, pp. 1273-1290, 1999.

[21] Vaudry C., et al, "Initiative mixte dans les DVP : de la pertinence a l'adaptation”. In Actes de l'atelier Documents Virtuels Personnalisables, Brest, 2002.

[22] Lionel Villard, et al, "An XML-based multimedia document processing model for content adaptation". In Proceeding of the Eighth International Conference on Digital Documents and Electronic Publishing (DDEP'00), 
International Journal Multimedia and Image Processing (IJMIP), Volume 2, Issues 1/2, March/June 2012

Springer Computer Science, Munich, Germany, pp. 104119, 2000.

[23] Wahl T., Rothermel K. "Representing Time in Multimedia Systems", IEEE Proceedings of the International Conference on Multimedia Computing and Systems. Boston, Massachusetts: IEEE Computer Society Press, pp. 538-543, 1994. 